\title{
Networked Conductive Polythiophene/Polyaniline Bottlebrushes with Modified Carbon Nanotubes As Hole Transport Layer in Organic Photovoltaics
}

\author{
LIANBING DENG, ${ }^{1,2,3}$ DAMING LI, ${ }^{1,2,4,6}$ and SAMIRA AGBOLAGHI $\oplus^{5,7}$ \\ 1.-Zhuhai Da Hengqin Science and Technology Development Co., Ltd, Hengqin New Area \\ 519031, Guangdong, China. 2.-Guangdong Qinzhi Science and Technology Research Institute, \\ Hengqin New Area 519031, Guangdong, China. 3.-Huazhong University of Science and \\ Technology, Wuhan 430074, Hubei, China. 4.-Institute of Data Science, City University of \\ Macau, Macau 999078, China. 5.-Chemical Engineering Department, Faculty of Engineering, \\ Azarbaijan Shahid Madani University, P.O. Box 5375171379, Tabriz, Iran. 6.-e-mail: \\ dmli@cityu.mo. 7.—e-mail: s.agbolaghi@azaruniv.ac.ir
}

Three types of hole transport layers (HTLs) were developed based on multiwalled carbon nanotubes (CNTs), including pure CNT thin films, CNT:poly(3thiophene ethanol) (P3ThEt)-g-polyaniline (PANI) nanocomposites, and CNTg-poly(3-dodecyl thiophene) (PDDT):P3ThEt-g-PANI interconnected networks, and utilized in poly[benzodithiophene-bis(decyltetradecyl-thien) naphthothiadiazole] (PBDT-DTNT):phenyl-C61-butyric acid methyl ester (PC61BM) and poly[bis(triiso-propylsilylethynyl) benzodithiophene-bis(decyltetradecyl-thien) naphthobisthiadiazole] (PBDT-TIPS-DTNT-DT):PC61BM solar cells. Pure CNTs were not the appropriate candidates for application instead of conventional poly(3,4-ethylenedioxythiophene):poly(styrenesulfonate) (PEDOT:PSS) HTLs. To tackle this issue, the CNT:P3ThEt-g-PANI and CNT-g-PDDT:P3ThEt-g-PANI films were focused with thicknesses of $10 \mathrm{~nm}$ and $20 \mathrm{~nm}$. The prominent characteristics peaked at $20-\mathrm{nm}$ thin films of CNT-g-PDDT:P3ThEt-g-PANI, demonstrating the largest power conversion efficiencies (PCE) of $5.65\left(12.84 \mathrm{~mA} / \mathrm{cm}^{2}, 62 \%\right.$, and $\left.0.71 \mathrm{~V}\right)$ and $4.80 \%$ $\left(11.59 \mathrm{~mA} / \mathrm{cm}^{2}, 60 \%\right.$, and $\left.0.69 \mathrm{~V}\right)$ in the BDT-DTNT and PBDT-TIPS-DTNTDT based devices, respectively. The CNT-g-PDDT:P3ThEt-g-PANI thin films which possess an interconnected network, composed of grafted-CNTs and P3ThEt-g-PANI bottlebrushes, were proper alternatives for conventional PEDOT:PSS HTLs and warranted the superior photovoltaic results by smooth morphologies (root mean square $=1.0-1.1 \mathrm{~nm}$ ) and low sheet resistance $(2.2-$ $8.3 \times 10^{4} \Omega / \mathrm{sq}$ ). The corresponding systems without grafting of CNT precursors were the second categories of well-functioned HTLs $(3.13-4.04 \%)$ and had somehow decreased physical and photovoltaic properties. 


\section{Graphic Abstract}

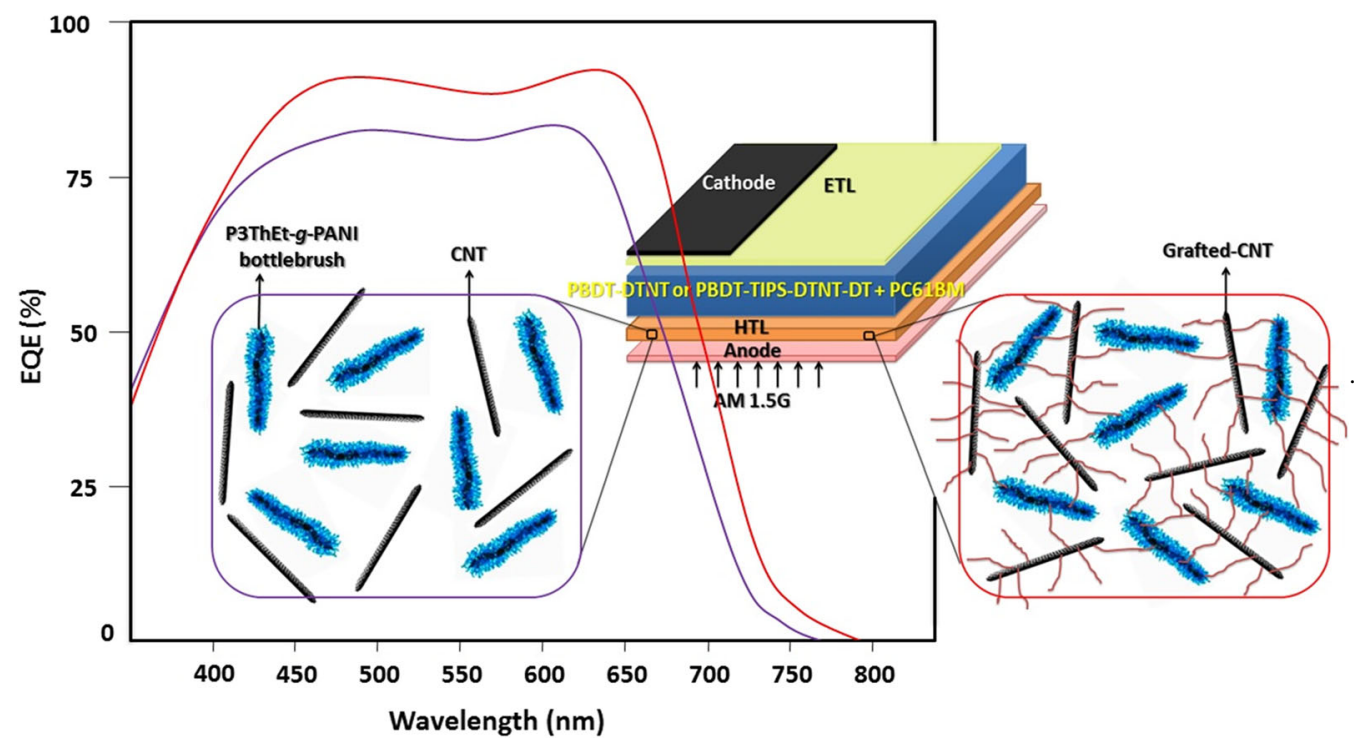

Key words: HTL, CNT, polythiophene, PANI, PCE

\section{INTRODUCTION}

Since demand has increased for renewable energies, solar cells have become a most promising candidate as a future energy source. Polymer solar cells have recently attracted great attention as a potential alternative for the conventional siliconbased photovoltaics. Advantages of polymer solar cells consist of their low cost, ease of fabrication, and their potential for flexible and large area devices. ${ }^{1,2}$ A conspicuous progress in the performance of polymeric photovoltaics has been achieved by introducing the donor-acceptor bulk heterojunction (BHJ) structures in the active layers. ${ }^{3-6}$ In polymer solar cells, the power conversion efficiency (PCE) was much increased when poly(3,4-ethylenedioxythiophene):poly(styrenesulfonate) (PEDOT:PSS) was introduced as a hole transport layer (HTL) by lowering the contact resistance and leakage current. ${ }^{7-9}$ Although the performances of polymeric photovoltaics have been elevated by means of PEDOT:PSS layers, ${ }^{10-12}$ distinct problems have been reported for the systems in question. Since PEDOT:PSS is dispersed in water with large particle sizes, the aggregates of particles may induce some defects and thus degradation to the device. ${ }^{13}$ Thanks to the strong acidic nature of PSS, it may degrade the indium tin oxide (ITO) surface, and thereby deteriorate the solar cell performance and long-term stability. ${ }^{14-17}$ In addition, the morphology and conductivity of spin-coated PEDOT:PSS films vary over different film regions, leading to a heterogeneous charge extraction. ${ }^{18,19}$ Therefore, some effort has been devoted to replace the conventional HTL in the organic photovoltaics. ${ }^{20-22}$

Copper(II) bromide $\left(\mathrm{CuBr}_{2}\right)$ salt has been applied to dope the PEDOT:PSS as the HTL in polymer solar cells in order to improve the conductivity of PEDOT:PSS film, and thereby the device efficiency ${ }^{23}$ Replacing the PEDOT:PSS with vanadium pentoxide $\left(\mathrm{V}_{2} \mathrm{O}_{5}\right)$ in the poly[[4,8-bis[(2-ethylhexyl)oxy]benzo[1,2-b:4,5-b']dithiophene-2,6-

diyl][3-fluoro-2-[(2-ethylhexyl)carbonyl]thieno[3,4-

b]thiophenediyl]] (PTB7)-based photovoltaics led to the simultaneous improvement in all photovoltaic characteristics, in particular PCE (7.03\%). ${ }^{24}$ Application of $\mathrm{V}_{2} \mathrm{O}_{5} \cdot n \mathrm{H}_{2} \mathrm{O}$ film as HTL instead of PEDOT:PSS in the polymer solar cells resulted in highly efficient systems with low-cost and massive production. ${ }^{25} \mathrm{~A}$ method was also proposed for developing a low-temperature solution-processed vanadium oxide and PSS composite to act as an efficient HTL in the organic photovoltaics. ${ }^{26}$ In another work, molybdenum trioxide $\left(\mathrm{MoO}_{3}\right)$ HTLs were exposed to different environments such as oxygen, light, and humidity to investigate different parameters within the HTL and the reactions at interfaces. ${ }^{27}$ Solution processable self-doped sulfonic acid ring-substituted polyaniline thin film was also employed as the HTL in the inverted solar 
cells. ${ }^{28}$ Moreover, water-soluble hydrochloric acid doped polyanilines were utilized as $p$-type electrode buffer layer (EBL) in high-efficient polymer solar cells and the PCE of 9\% was acquired. The excellent performances were attributed to high transparency, small film roughness and suitable work function. ${ }^{29}$ The graft copolymers such as PSS grafted with polyaniline (PSS-g-PANI) ${ }^{30,31}$ and polypyrrole (PSS$g$-PPY $)^{32}$ were also used as HTLs in the polymer solar cells. The PSS-g-PANI films possessing superior electrochemical stability compared with PEDOT:PSS films reflected a $20 \%$ larger efficiency. ${ }^{30} \mathrm{~A}$ series of cross-linkable hole transport materials subsuming the indacenodithiophene, bithiophene, and thiophene units functionalized by two triarylamine groups have been designed as new anode buffer layers. ${ }^{33}$ Kymakis et al. ${ }^{34}$ reported the use of surfactant-free single-walled carbon nanotube (SWCNT) films, prepared by spin-coating, as the HTL in the BHJ solar cells. ${ }^{34}$ The graphene oxide HTLs were also applied in the large area high efficiency organic solar cells. ${ }^{35}$

Two types of $p$-type EBLs including the multiwalled CNTs (MWCNTs):poly(3-thiophene ethanol) (P3ThEt)-g-polyaniline (PANI) and CNT-g-poly(3-dodecyl thiophene) (PDDT):P3ThEt-g-PANI thin films were designed and utilized in the poly[benzodithiophene-bis(decyltetradecyl-thien) naphthothiadiazole] (PBDT-DTNT):phenyl-C61-butyric acid methyl ester (PC61BM) and poly[bis(triiso-propylsilylethynyl) benzodithiophene-bis(decyltetradecyl-thien) naphthobisthiadiazole] (PBDT-TIPS-DTNT-DT):PC61BM solar cells. The prominent characteristics peaked at the $20 \mathrm{~nm}$ thin films of CNT-g-PDDT:P3ThEt-g-PANI, demonstrating the largest PCEs of $5.65(12.84 \mathrm{~mA} /$ $\mathrm{cm}^{2}, 62 \%$, and $\left.0.71 \mathrm{~V}\right)$ and $4.80 \%\left(11.59 \mathrm{~mA} / \mathrm{cm}^{2}, 60 \%\right.$, and $0.69 \mathrm{~V}$ ) in the BDT-DTNT and PBDT-TIPSDTNT-DT based devices, respectively.

\section{EXPERIMENTAL}

\section{Synthesis and Characterization of P3ThEt-g-PANI}

The synthesis of P3ThEt and phenylamine-functionalized P3ThEt with $p$-anthranilice acid (PhAP3ThEtM) are reported in Supplementary Information. A proper reactor was loaded with dimethylformamide (DMF) and $1.0 \mathrm{~g}$ PhAP3ThEtM. The oxidant solution [ammonium persulfate (APS) in $\left.\mathrm{H}_{2} \mathrm{SO}_{4}(0.5 \mathrm{M})\right]$ was added to the system and the temperature was switched to $0^{\circ} \mathrm{C}$. The aniline monomer was separately dissolved in $\mathrm{H}_{2} \mathrm{SO}_{4}$ and added to the solution of oxidant and PhAP3ThEtM. The mixture was stirred for $10 \mathrm{~h}$ at $0^{\circ} \mathrm{C}$ and, subsequently, the polymer was filtered, washed with water/ methanol, and dried in vacuum. The chemical structure of P3ThEt-g-PANI bottlebrushes is depicted in Fig. 1a. FT-IR spectra of P3ThEt and P3ThEtM are illustrated in Fig. S1(a). Figure 1a also represents FT-IR spectrum of P3ThEt-g-PANI bottlebrushes. FT-IR of P3ThEt-g-PANI bottlebrushes exhibited the prominent peaks for stretching vibrations of aromatic $\mathrm{C}-\mathrm{H}\left(3050-3200 \mathrm{~cm}^{-1}\right), \gamma(\mathrm{C}-\mathrm{H})$ in the aromatic ring $\left(757 \mathrm{~cm}^{-1}\right.$ and $\left.794 \mathrm{~cm}^{-1}\right)$, the $\mathrm{N}-\mathrm{H}$ stretching vibration $\left(3456 \mathrm{~cm}^{-1}\right)$, stretching vibration of the $\mathrm{C}=\mathrm{C}$ in the benzenoid units $\left(1577 \mathrm{~cm}^{-1}\right)$, aromatic $\mathrm{C}-\mathrm{N}$ stretching vibration $\left(1307 \mathrm{~cm}^{-1}\right)$ and weak aromatic bands $\left(1650-1900 \mathrm{~cm}^{-1}\right){ }^{36,37}$ The considerable variations in these spectra included the stretching vibrations of aliphatic C-H at $2800-2950 \mathrm{~cm}^{-1}$ and the N$\mathrm{H}$ stretching vibrations at $3324 \mathrm{~cm}^{-1}$. Notably, other peaks of P3ThEt overlapped with the sharp PANI bands (Fig. 1a). ${ }^{1}$ HNMR spectra of P3ThEt and P3ThEtM are shown in Fig. S1(b) and (c), respectively. The gel permeation chromatography (GPC, Agilent 1100) traces of P3ThEt and P3ThEt-g-PANI are illustrated in Fig. 1b. The GPC chromatograms confirmed that the polymeric materials were synthesized with the narrow distributions. The data details of GPC measurements are also reported in Table SI. The electroactivity of P3ThEt, P3ThEt-g-PANI, and H-PANI was studied by cyclic voltammetric (CV) measurements at $25 \mathrm{mV}$ and scan rates of $10-50 \mathrm{mV} /$ $\mathrm{s}$ in $\mathrm{H}_{2} \mathrm{SO}_{4}(1 \mathrm{M})$ versus the reference electrode ( $\mathrm{Ag} /$ $\mathrm{AgCl})$ (Figs. S2(a-f)). The electrical conductivities (Azar Electrode, Urmia, Iran) are also reported in Table SII. The highest conductivity of $0.32 \mathrm{~S} / \mathrm{cm}$ was recorded for the pristine P3ThEt backbones. The conductivity of P3ThEt-g-PANI bottlebrushes was equal to $0.27 \mathrm{~S} / \mathrm{cm}$. On the basis of field emission scanning electron microscopic (FESEM) analysis [type 1430 VP (LEO Electron Microscopy Ltd, Cambridge, UK)], bubble-like nanostructures were detected for the assemblies of P3ThEt-g-PANI bottlebrushes (Fig. 1c). The atomic force microscopic (AFM) image of P3ThEt-g-PANI bottlebrushes is also depicted in Fig. 1d for elucidating the morphology of PANI-covered P3ThEt backbones.

\section{Synthesis of PBDT-DTNT, PBDT-TIPS-DTNT-DT, and Grafted-CNTs}

The PBDT-DTNT and PBDT-TIPS-DTNT-DT conjugated polymers were synthesized via stillecoupling. ${ }^{38,39}$ More details are represented in Supplementary Information (Figs. S3-S5). The preparation steps of carboxylated CNT (CNT-COOH), thiophene functionalized CNT (CNT-functionalizedCOOTh), and the chemical oxidative graft polymerization of 3-dodecylthiophene from CNT [CNT-gpoly(3-dodecyl thiophene) (PDDT)] are also represented in Supplementary Information (Fig. S6).

\section{Cell Fabrication and Characterization}

The photovoltaic devices were fabricated with a configuration of ITO/HTL/PBDT-DTNT or PBDTTIPS-DTNT-DT:PC61BM/LiF/Al. The HTLs were separately composed of PEDOT:PSS, CNT, CNT:P3ThEt-g-PANI and CNT-g-PDDT:P3ThEt-gPANI thin films with thicknesses of $10 \mathrm{~nm}, 20 \mathrm{~nm}$, and $50 \mathrm{~nm}$. For this purpose, a mixture of CNT or CNT-g-PDDT:P3ThEt-g-PANI with a weight ratio 


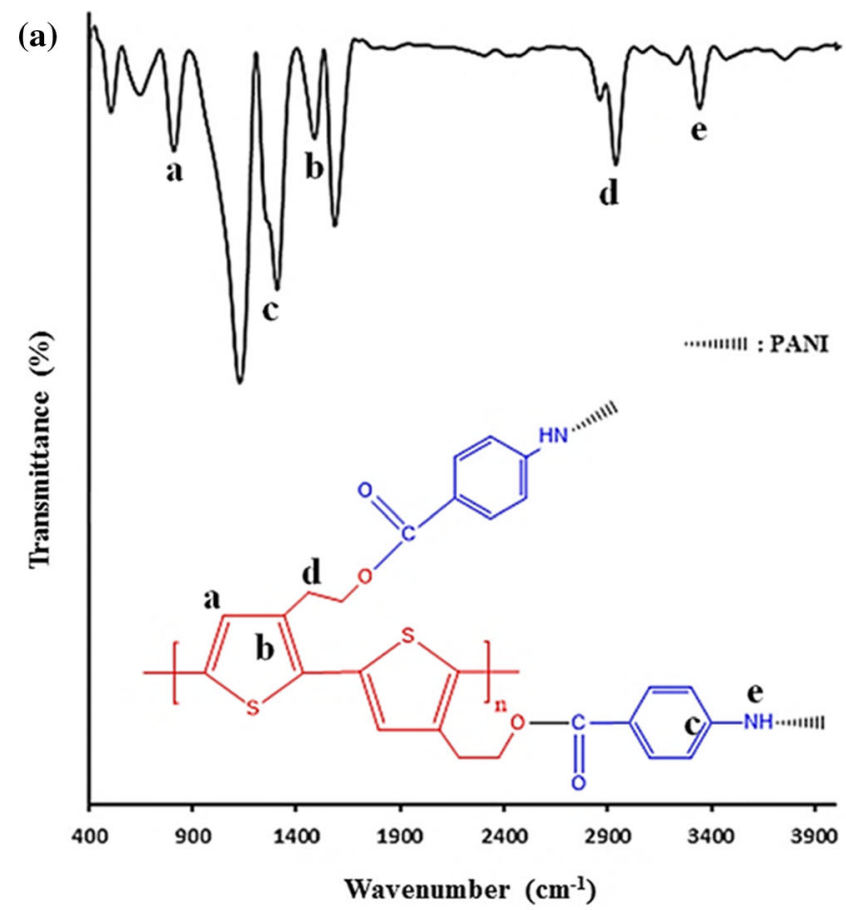

(b)
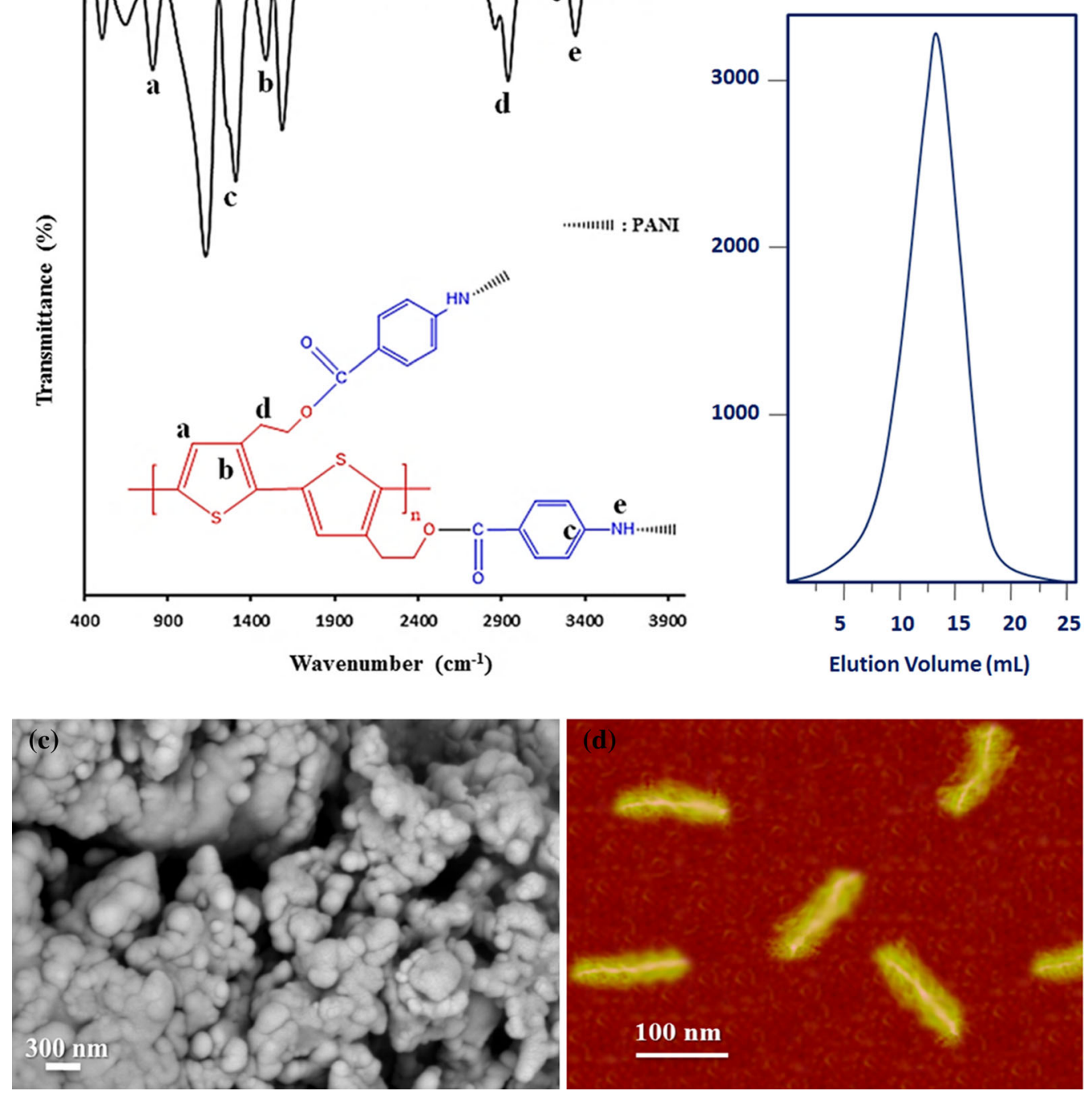

Fig. 1. Chemical structure and FT-IR spectrum (a), GPC trace in DMF (b), FESEM (c) and AFM (d) images of P3ThEt-g-PANI bottlebrushes.

of $1: 1$ was dispersed in dichloromethane at $10^{-3} \mathrm{mg} /$ $\mathrm{mL}$ and shaking continued for $15 \mathrm{~h}$. Afterward, the resultant product was centrifuged at $6000 \mathrm{rpm}$ $(6 \mathrm{~h})$. After $15 \mathrm{~h}$ of shaking and $6 \mathrm{~h}$ of centrifuging, the result comprised to some extent homogeneous solutions. Moreover, the spin-coating was carried out upon completion of centrifuge step. Therefore, the weight ratio of $1: 1$ was partly retained for the CNT or CNT-g-PDDT:P3ThEt-g-PANI compositions. In addition, the method in question was repeated several times for the fabrication of solar cells, and similar results with a high accuracy were obtained. The CNT precursors: P3ThEt-g-PANI films were then spin-coated from the prepared solution onto the ITO/glass substrate. The weight ratio of PBDT-DTNT or PBDT-TIPS-DTNTDT:PC61BM was also fixed at 1:1 in chlorobenzene with a concentration of $25 \mathrm{mg} / \mathrm{mL}$. The thicknesses of hole transport and active layers were $(10 \mathrm{~nm}$ and $20 \mathrm{~nm}$ ) and (150 nm) [surface profilometer (Tencor, Alpha-500)], respectively. A $\mathrm{LiF}$ interfacial layer
$(1 \mathrm{~nm})$ and a $80 \mathrm{~nm}$ film of $\mathrm{Al}$ were subsequently coated onto the device under $2 \times 10^{-4} \mathrm{~Pa}$. The current density-voltage $(\mathrm{J}-\mathrm{V})$ characterization of the photovoltaic cells was conducted using a computer-controlled measurement unit from Newport under the illumination of AM1.5G, $100 \mathrm{~mW} / \mathrm{cm}^{2}$.

\section{RESULTS AND DISCUSSION}

The three types of HTLs were prepared based on the CNT precursors including the pure CNT thin films, the CNT:P3ThEt-g-PANI nanocomposites, and the CNT-g-PDDT:P3ThEt-g-PANI interconnected networks. These $p$-type EBLs were employed with thicknesses of $10 \mathrm{~nm}$ and $20 \mathrm{~nm}$ to reach optimal conditions. The bare ITO and ITO:PEDOT:PSS $(50 \mathrm{~nm})$ samples were also prepared to compare with the principal CNT-based systems. A typical configuration for the solar cells fabricated in the current work is displayed in the middle panel of Fig. 2. The active layers of all constructed 


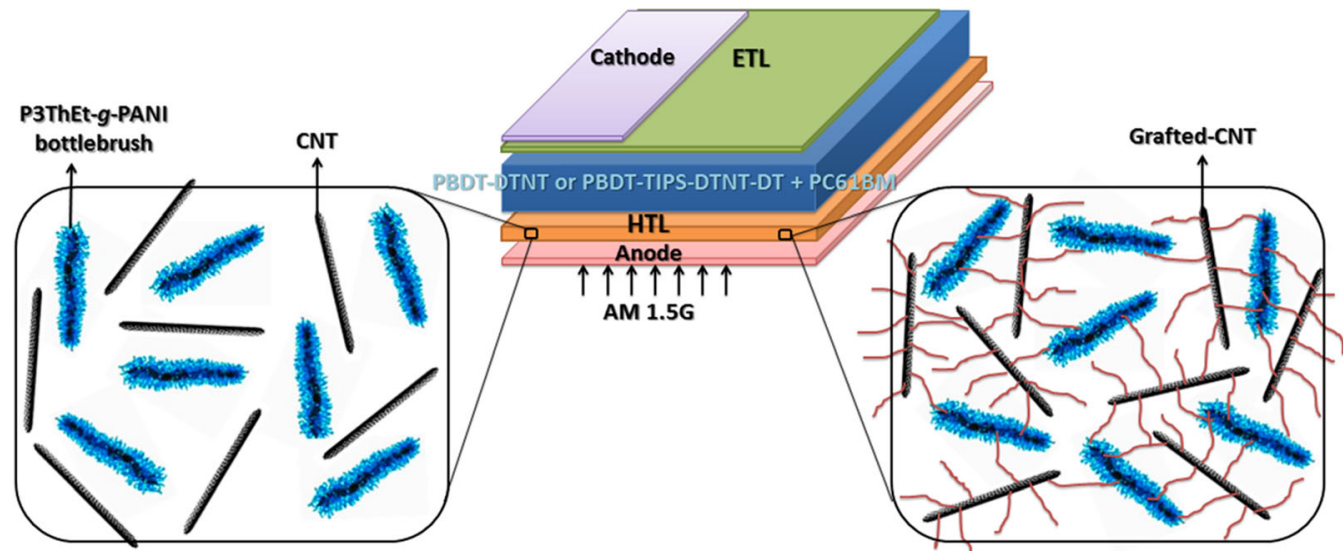

Fig. 2. Scheme of a typical photovoltaic cell having ITO/HTL/PBDT-DTNT or PBDT-TIPS-DTNT-DT:PC61BM/LiF/Al structure with enlarged CNT:P3ThEt-g-PANI (left) and CNT-g-PDDT:P3ThEt-g-PANI (right) HTLs.
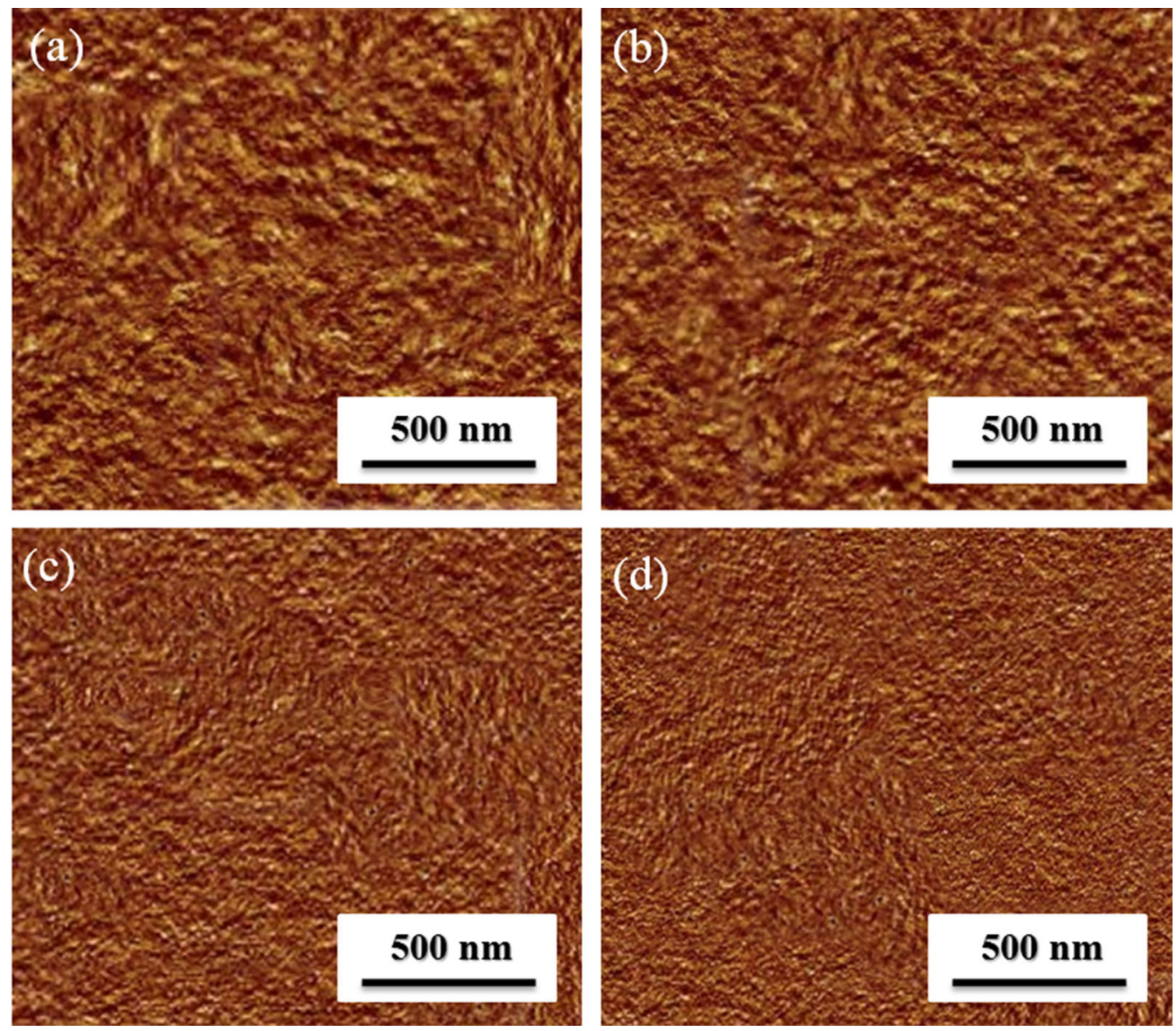

Fig. 3. AFM phase images of (a) PEDOT:PSS (50 nm), (b) CNT (20 nm), (c) CNT:P3ThEt- $g$-PANI (20 nm) and (d) CNT- $g$-PDDT:P3ThEt- $g$ PANI $(20 \mathrm{~nm})$ thin films.

photovoltaic devices were composed of PBDTDTNT:PC61BM and PBDT-TIPS-DTNTDT:PC61BM constituents. The enlarged schemes of CNT:P3ThEt-g-PANI and CNT-g-PDDT:P3ThEt$g$-PANI HTLs are depicted in the left and right panels of Fig. 2, respectively. Mainly, 16 different types of devices were fabricated and investigated in this work to clarify the effect of each HTL on the functions of PBDT-DTNT:PC61BM and PBDTTIPS-DTNT-DT:PC61BM photovoltaics.

The scanning probe microscope (SPM, Nanoscope) was applied to record the phase images in tapping mode. The SPM phase contrast images of PEDOT:PSS, CNT, CNT:P3ThEt-g-PANI and CNT-gPDDT:P3ThEt- $g$-PANI thin films are represented in Fig. 3a, b, c, and d, respectively. The PEDOT:PSS 
and CNT HTLs had the larger phase contrast with respect to the CNT:P3ThEt-g-PANI and CNT-gPDDT:P3ThEt-g-PANI ones. A conspicuous phase separation in PEDOT:PSS reflected an inhomogeneous hole collection for photovoltaic device, ${ }^{19}$ and thereby a degradation in the cell performance. Among the smooth morphologies, the higher conductivity of CNT-g-PDDT:P3ThEt-g-PANI (0.29 S/ $\mathrm{cm}$ versus $0.18 \mathrm{~S} / \mathrm{cm}$ for CNT:P3ThEt-g-PANI) made it the best choice for HTL.

The transmittance spectra of PEDOT:PSS, CNT, CNT:P3ThEt-g-PANI and CNT-g-PDDT:P3ThEt-gPANI $p$-type EBLs spin-coated on the glass substrates are illustrated in Fig. 4 . The transmittance details are also tabulated in Table I. The most transparent HTL was the PEDOT:PSS (94-97\%) having a thickness of $50 \mathrm{~nm}$. The most similar thin film to the conventional PEDOT:PSS from the perspective of transparency was the thinner pure CNT HTL with a thickness of $10 \mathrm{~nm}$ (93-96\%). A declining trend was detected in the transmittance versus the HTL thickening. Therefore, the thinner CNT, CNT:P3ThEt-g-PANI, and CNT-gPDDT:P3ThEt-g-PANI EBLs allowed a higher photon flux to reach the PBDT-DTNT:PC61BM and PBDT-TIPS-DTNT-DT:PC61BM active layers for the next photon-to-current procedure. The thicker pure CNT thin film $(20 \mathrm{~nm})$ represented the second high-quality transmittance (88-94\%), as shown in Fig. 4. Interestingly, the CNT:P3ThEt-g-PANI nanocomposites and the CNT-g-PDDT:P3ThEt-g-
PANI networks were more opaque with respect to the pure CNT thin films in similar thicknesses. In fact, the presence of polythiophenic and polyanilinic constituents induced a higher opacity on the mentioned films. The transmittance percentages of CNT:P3ThEt-g-PANI $\quad(10 \mathrm{~nm}), \quad$ CNT:P3ThEt-gPANI $\quad(20 \mathrm{~nm}), \quad$ CNT-g-PDDT:P3ThEt-g-PANI $(10 \mathrm{~nm})$ and CNT-g-PDDT:P3ThEt-g-PANI $(20 \mathrm{~nm})$ were equal to $85-91 \%, 82-87 \%, 84-90 \%$ and $80-$ $85 \%$, respectively. Moreover, in completely similar conditions, the CNT-g-PDDT:P3ThEt-g-PANI thin films were slightly more opaque compared to those composed of the ungrafted CNT precursors (CNT:P3ThEt-g-PANI). By regarding the transmittance spectra in Fig. 4, it can be understood that the film thickness was a more effective parameter than the CNT grafting on the HTL transmittance.

In addition to the transmittance measurements, the sheet resistance and roughness parameters were recorded for the prepared HTLs for further elucidating the proper candidates for the conventional PEDOT:PSS replacement. The root mean square (RMS) and sheet resistance values are reported in Table I for the PEDOT:PSS $(50 \mathrm{~nm})$, CNT (10 nm and $20 \mathrm{~nm}$ ), CNT:P3ThEt-g-PANI $(10 \mathrm{~nm}$ and $20 \mathrm{~nm})$ and CNT-g-PDDT:P3ThEt-gPANI (10 $\mathrm{nm}$ and $20 \mathrm{~nm}$ ) HTLs. A sheet resistance of $3.9 \times 10^{6} \Omega / \mathrm{sq}$ was determined for the $50 \mathrm{~nm}$ PEDOT:PSS thin film, using the four-point probe method. The sheet resistance values of all designed $p$-type EBLs were less than that detected in the

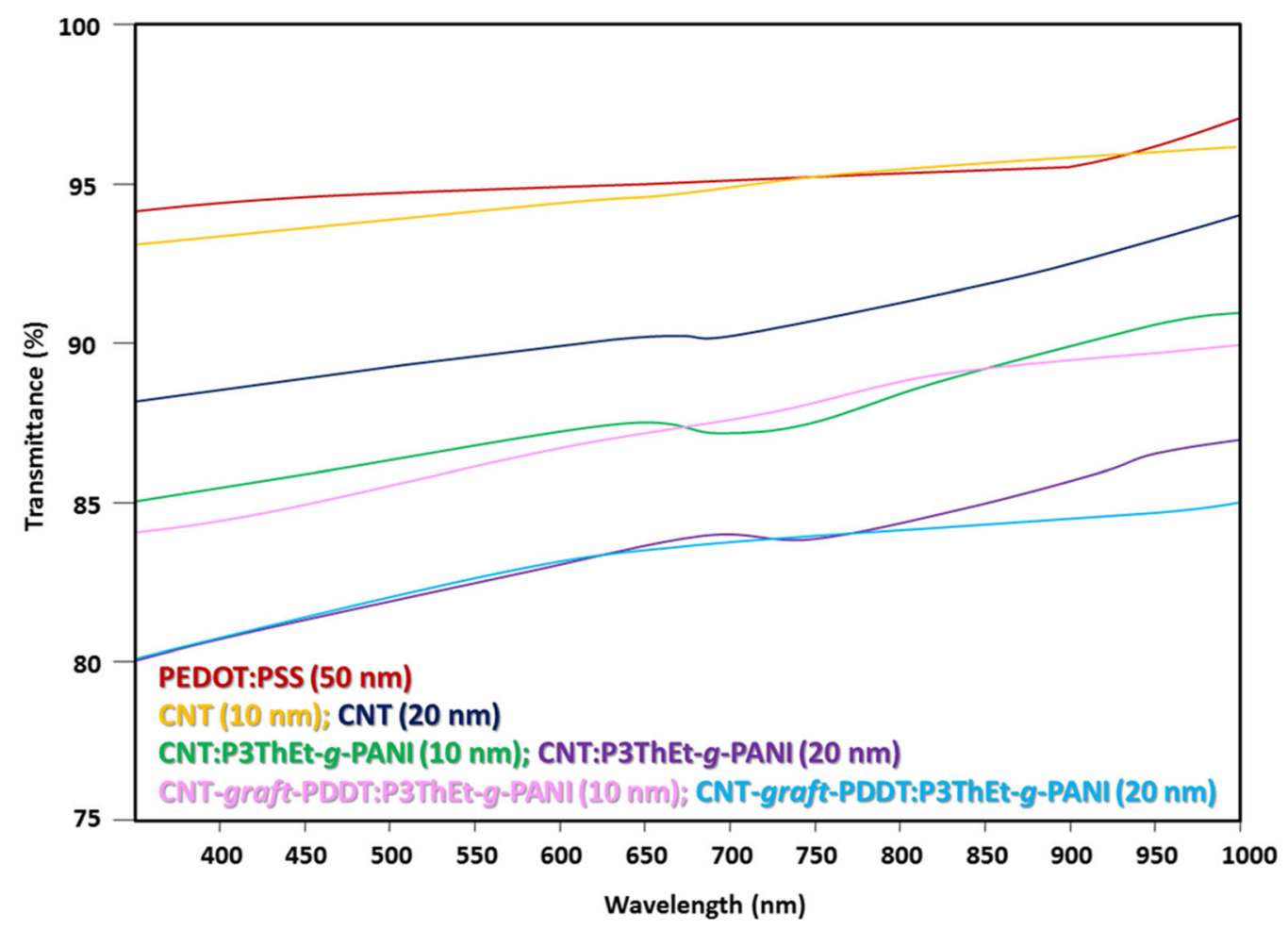

Fig. 4. Transmittance spectra of PEDOT:PSS, CNT, CNT:P3ThEt-g-PANI and CNT-g-PDDT:P3ThEt-g-PANI HTLs with different thicknesses spin-coated on the glass substrates. 
Table I. Physical characteristics of PEDOT:PSS, CNT, CNT:P3ThEt-g-PANI and CNT-g-PDDT:P3ThEt-gPANI thin films with different thicknesses

\begin{tabular}{|c|c|c|c|c|}
\hline Sample & Thickness (nm) & Transmittance $(\%)$ & RMS (nm) & Sheet resistance $(\Omega /$ sq $)$ \\
\hline PEDOT:PSS & 50 & $94-97$ & 1.5 & $3.9 \times 10^{6}$ \\
\hline CNT & 10 & $93-96$ & 1.7 & $8.5 \times 10^{5}$ \\
\hline CNT & 20 & $88-94$ & 2.8 & $6.7 \times 10^{5}$ \\
\hline CNT:P3ThEt-g-PANI & 10 & $85-91$ & 1.4 & $4.9 \times 10^{5}$ \\
\hline CNT:P3ThEt-g-PANI & 20 & $82-87$ & 1.3 & $1.1 \times 10^{5}$ \\
\hline CNT-g-PDDT:P3ThEt-g-PANI & 10 & $84-90$ & 1.1 & $8.3 \times 10^{4}$ \\
\hline CNT-g-PDDT:P3ThEt-g-PANI & 20 & $80-85$ & 1.0 & $2.2 \times 10^{4}$ \\
\hline
\end{tabular}

conventional PEDOT:PSS film. The lowest sheet resistance $\left(2.2 \times 10^{4} \Omega / \mathrm{sq}\right)$ was recorded for the CNT-g-PDDT:P3ThEt-g-PANI $(20 \mathrm{~nm})$ thin film, which was the most opaque system (80-85\%). From the same order of magnitude, the thinner CNT-gPDDT:P3ThEt-g-PANI HTL $(10 \mathrm{~nm})$ possessed a sheet resistance of $8.3 \times 10^{4} \Omega / \mathrm{sq}$. An increasing trend was observed in the sheet resistance values with a decrease in the thin film thicknesses. This phenomenon was detected in all types of HTLs including CNT, CNT:P3ThEt-g-PANI, and CNT-gPDDT:P3ThEt-g-PANI. In the CNT-gPDDT:P3ThEt-g-PANI thin films, the interactions between the polythiophenic and polyanilinic grafts may facilitate the interconnected networking of different constituents, thereby the smoothest morphologies $(\mathrm{RMS}=1.0-1.1 \mathrm{~nm})$ with the smallest sheet resistances $\left(2.2-8.3 \times 10^{4} \Omega / \mathrm{sq}\right)$ were reached.

The chemically simpler nanostructures without any grafting of CNT precursors, i.e., CNT:P3ThEt$g$-PANI represented the larger sheet resistances $\left(1.1 \times 10^{5} \Omega / \mathrm{sq}\right.$ in $20 \mathrm{~nm}$ and $4.9 \times 10^{5} \Omega / \mathrm{sq}$ in $10 \mathrm{~nm}$ thicknesses) and the rougher thin films $(1.3 \mathrm{~nm}$ in $20 \mathrm{~nm}$ and $1.4 \mathrm{~nm}$ in $10 \mathrm{~nm}$ thicknesses). The role of PDDT grafts onto the CNTs was probably smoothing the thin films by filling the voids. Among the CNT-based $p$-type EBLs, the pure CNT films, without any blending with the P3ThEtg-PANI rod-rod bottlebrushes and also surface modification via grafting with the polythiophenic chains, led to the largest sheet resistance $\left(6.7 \times 10^{5} \Omega / \mathrm{sq}\right.$ in $20 \mathrm{~nm}$ and $8.5 \times 10^{5} \Omega / \mathrm{sq}$ in $10 \mathrm{~nm}$ thicknesses) and RMS $(2.8 \mathrm{~nm}$ in $20 \mathrm{~nm}$ and $1.7 \mathrm{~nm}$ in $10 \mathrm{~nm}$ thicknesses) values. As previously shown by the SPM measurements, the PEDOT:PSS $(\mathrm{RMS}=1.5 \mathrm{~nm})$ and pure CNT $(\mathrm{RMS}=1.7-2.8 \mathrm{~nm})$ had the larger phase contrasts compared with the modified CNT:P3ThEt-g-PANI (RMS $=1.3-1.4 \mathrm{~nm})$ and CNT-g-PDDT:P3ThEt-gPANI $(\mathrm{RMS}=1.0-1.1 \mathrm{~nm})$ HTLs. Actually, an interconnected network composed of grafted-CNTs and P3ThEt- $g$-PANI bottlebrushes was developed in the well-functioned smooth CNT-g-PDDT:P3ThEtg-PANI thin films which reflected the low sheet resistance and other qualified characteristics after being included as the HTLs in the PBDT-
DTNT:PC61BM and PBDT-TIPS-DTNTDT:PC61BM solar cells. The second categories of well-acted systems were those prepared on the basis of CNT:P3ThEt-g-PANI thin films with the larger sheet resistances and the rougher morphologies.

For further investigating the impact of each HTL on the functions of ultimate photovoltaic devices, the 16 different devices were fabricated using the seven distinct $p$-type EBLs. The current density $(J)$ voltage $(V)$ curves were reported in Fig. $5 \mathrm{a}$ and $\mathrm{b}$. The data details are also tabulated in Table II. So as to demonstrate the accuracy of reported data, the standard deviation (SD) and average values (Avg) are also included in Table II. The primary ITO:PBDT-DTNT:PC61BM and ITO:PBDT-TIPSDTNT-DT:PC61BM devices without any HTLs represented the lowest short circuit current density $\left(J_{\mathrm{sc}}\right)$, fill factor $(\mathrm{FF})$, open circuit voltage $\left(V_{\text {oc }}\right)$ and $\mathrm{PCE}$. The data in question were $\left(6.89 \mathrm{~mA} / \mathrm{cm}^{2}\right.$ and $\left.6.03 \mathrm{~mA} / \mathrm{cm}^{2}\right),(48 \%$ and $46 \%),(0.56 \mathrm{~V}$ and $0.55 \mathrm{~V})$ and $(1.85 \%$ and $1.52 \%)$ for the PBDT-DTNT and PBDT-TIPS-DTNT-DT based systems, respectively. The insertion of a 50-nm PEDOT:PSS HTL in these photovoltaics significantly improved the cell features, i.e., $\left(9.29 \mathrm{~mA} / \mathrm{cm}^{2}\right.$ and $\left.8.80 \mathrm{~mA} / \mathrm{cm}^{2}\right),(53 \%$ and $52 \%),(0.65 \mathrm{~V}$ and $0.65 \mathrm{~V})$ and $(3.20 \%$ and $2.97 \%)$. That is to say, in all types of $p$-type EBLs, the photovoltaic properties were better for the PBDT-DTNT:PC61BM active layers in comparison to the PBDT-TIPS-DTNT-DT:PC61BM ones, originating from the chemical structure and charge transporting aspects of the conjugated polymers.

The replacement of PEDOT:PSS $(50 \mathrm{~nm})$ with the pure CNT films having a thickness of $10 \mathrm{~nm}$ did not afford the characteristics as high as those detected in the ITO:PEDOT:PSS (50 nm) systems. Efficacies of $2.84 \%\left(8.84 \mathrm{~mA} / \mathrm{cm}^{2}, 51 \%\right.$, and $\left.0.63 \mathrm{~V}\right)$ and $2.33 \%$ $\left(7.66 \mathrm{~mA} / \mathrm{cm}^{2}, 49 \%\right.$, and $\left.0.62 \mathrm{~V}\right)$ were determined in the ITO:CNT (10 nm):PBDT-DTNT:PC61BM and ITO:CNT (10 nm):PBDT-TIPS-DTNT-DT:PC61BM devices, respectively. Although the transmittance of CNT $(10 \mathrm{~nm})$ film was comparable with and its sheet resistance was lower than those recorded for the PEDOT:PSS $(50 \mathrm{~nm})$ film, the rougher nature (RMS $=1.7 \mathrm{~nm}$ versus $1.5 \mathrm{~nm}$ ) may result in somewhat low-qualified systems. The thicker CNT layers further destroyed the cell parameters $\left[\left(7.25 \mathrm{~mA} / \mathrm{cm}^{2}\right.\right.$ 

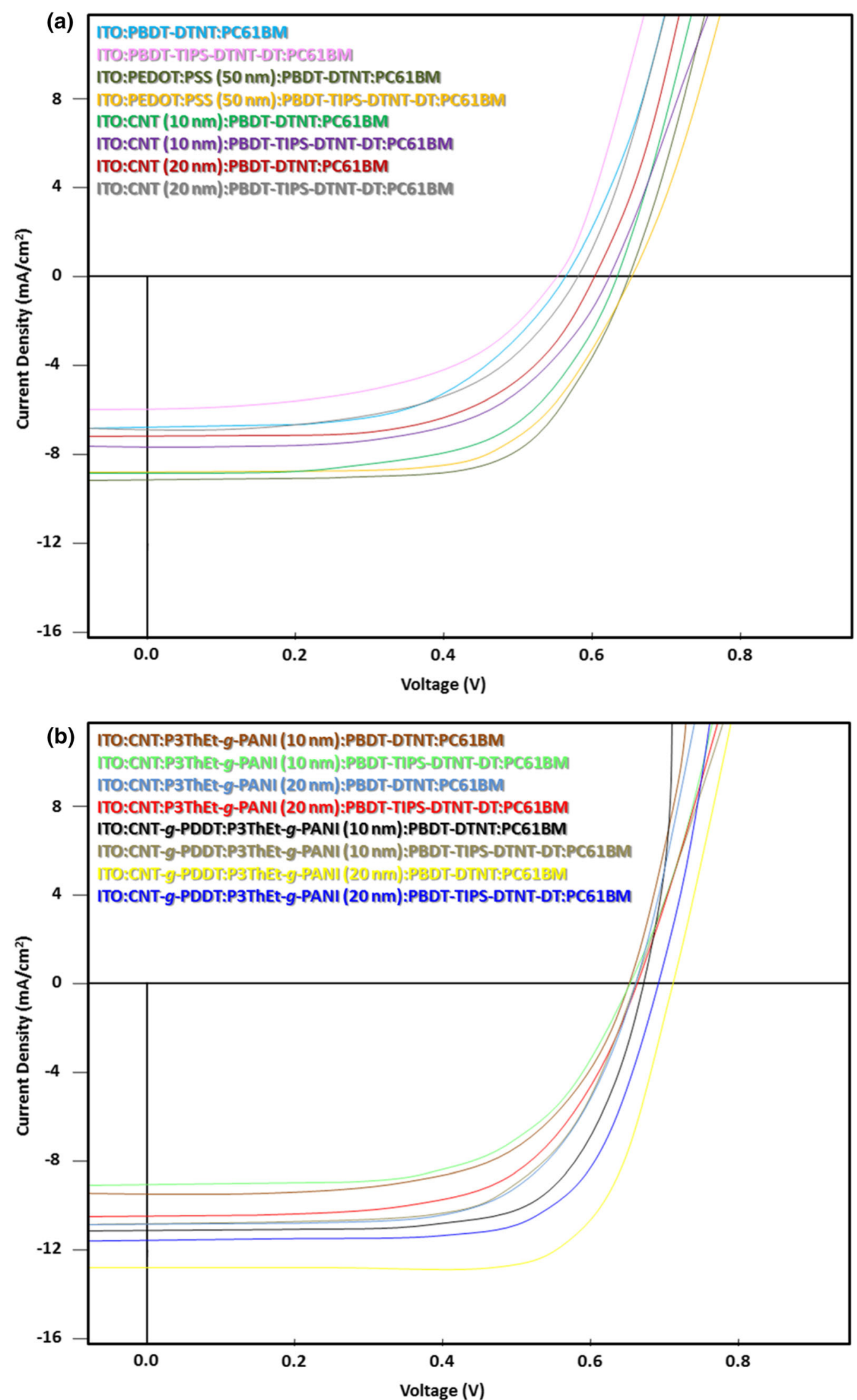

Fig. 5. J-V curves of BDT-DTNT or PBDT-TIPS-DTNT-DT:PC61BM photovoltaics subsuming the HTLs of PEDOT:PSS and CNT (a) as well as CNT:P3ThEt- $g$-PANI and CNT-g-PDDT:P3ThEt-g-PANI (b) versus the ITO only devices. 
Table II. Photovoltaic details for different solar cells

\begin{tabular}{|c|c|c|c|c|}
\hline Sample & $J_{\mathrm{sc}}\left(\mathbf{m A} / \mathbf{c m}^{2}\right)$ & FF (\%) & $V_{\mathbf{o c}}(\mathbf{V})$ & PCE (\%) \\
\hline ITO:PBDT-DTNT:PC61BM & $\begin{array}{c}\text { Avg: } 6.89 \text { (SD: } \\
0.05)\end{array}$ & $\begin{array}{c}\text { Avg: } 48 \text { (SD: } \\
0.51)\end{array}$ & $\begin{array}{c}\text { Avg: } 0.56(\mathrm{SD}: \\
0.010)\end{array}$ & $\begin{array}{c}\text { Avg: } 1.85 \text { (SD: } \\
0.09)\end{array}$ \\
\hline ITO:PBDT-TIPS-DTNT-DT:PC61BM & $\begin{array}{c}\text { Avg: } 6.03(\mathrm{SD}: \\
0.06)\end{array}$ & $\begin{array}{c}\text { Avg: } 46 \text { (SD: } \\
0.49)\end{array}$ & $\begin{array}{cl}\text { Avg: } & 0.55(\mathrm{SD}: \\
0.011)\end{array}$ & Avg: 1.52 (SD: \\
\hline $\begin{array}{l}\text { ITO:PEDOT:PSS (50 nm): } \\
\text { PBDT-DTNT:PC61BM }\end{array}$ & $\begin{array}{c}\text { Avg: } 9.29 \text { (SD: } \\
0.04)\end{array}$ & $\begin{array}{c}\text { Avg: } 53 \text { (SD: } \\
0.48)\end{array}$ & $\begin{array}{c}\text { Avg: } 0.65 \text { (SD: } \\
0.009)\end{array}$ & $\begin{array}{c}\text { Avg: } 3.20 \text { (SD: } \\
0.09)\end{array}$ \\
\hline $\begin{array}{l}\text { ITO:PEDOT:PSS (50 nm): } \\
\text { PBDT-TIPS-DTNT-DT:PC61BM }\end{array}$ & $\begin{array}{c}\text { Avg: } 8.80 \text { (SD: } \\
0.04)\end{array}$ & $\begin{array}{c}\text { Avg: } 52 \text { (SD: } \\
0.50)\end{array}$ & $\begin{array}{c}\text { Avg: } 0.65 \text { (SD: } \\
0.008)\end{array}$ & $\begin{array}{c}\text { Avg: } 2.97 \text { (SD: } \\
0.08)\end{array}$ \\
\hline $\begin{array}{l}\text { ITO:CNT }(10 \mathrm{~nm}): \text { PBDT- } \\
\text { DTNT:PC61BM }\end{array}$ & Avg: $\begin{array}{c}8.84(\mathrm{SD}: \\
0.05)\end{array}$ & $\begin{array}{l}\text { Avg: } 51 \text { (SD: } \\
0.45)\end{array}$ & $\begin{array}{c}\text { Avg: } 0.63 \text { (SD: } \\
0.006)\end{array}$ & $\begin{array}{c}\text { Avg: } 2.84(\mathrm{SD}: \\
0.09)\end{array}$ \\
\hline $\begin{array}{l}\text { ITO:CNT }(10 \mathrm{~nm}): \\
\text { PBDT-TIPS-DTNT-DT:PC61BM }\end{array}$ & $\begin{array}{c}\text { Avg: } 7.66(\mathrm{SD}: \\
0.06)\end{array}$ & $\begin{array}{c}\text { Avg: } 49 \text { (SD: } \\
0.43)\end{array}$ & $\begin{array}{c}\text { Avg: } 0.62 \text { (SD: } \\
0.007)\end{array}$ & $\begin{array}{c}\text { Avg: } 2.33(\mathrm{SD}: \\
0.07)\end{array}$ \\
\hline $\begin{array}{l}\text { ITO:CNT }(20 \mathrm{~nm}): \text { PBDT- } \\
\text { DTNT:PC61BM }\end{array}$ & Avg: $\begin{array}{c}7.25(\mathrm{SD}: \\
0.05)\end{array}$ & $\begin{array}{c}\text { Avg: } 49 \text { (SD: } \\
0.41)\end{array}$ & $\begin{array}{c}\text { Avg: } 0.60 \text { (SD: } \\
0.008)\end{array}$ & $\begin{array}{c}\text { Avg: } 2.13(\mathrm{SD}: \\
0.06)\end{array}$ \\
\hline $\begin{array}{l}\text { ITO:CNT (20 nm): } \\
\text { PBDT-TIPS-DTNT-DT:PC61BM }\end{array}$ & Avg: $\begin{array}{c}6.82(\mathrm{SD}: \\
0.07)\end{array}$ & $\begin{array}{l}\text { Avg: } 47 \text { (SD: } \\
0.44)\end{array}$ & $\begin{array}{c}\text { Avg: } 0.58(\mathrm{SD}: \\
0.009)\end{array}$ & $\begin{array}{c}\text { Avg: } 1.86(\mathrm{SD}: \\
0.08)\end{array}$ \\
\hline $\begin{array}{l}\text { ITO:CNT:P3ThEt-g-PANI }(10 \mathrm{~nm}) \text { : } \\
\text { PBDT-DTNT:PC61BM }\end{array}$ & $\begin{array}{c}\text { Avg: } 9.50(\mathrm{SD}: \\
0.06)\end{array}$ & $\begin{array}{c}\text { Avg: } 54 \text { (SD: } \\
0.39)\end{array}$ & $\begin{array}{c}\text { Avg: } 0.65 \text { (SD: } \\
0.007)\end{array}$ & $\begin{array}{c}\text { Avg: } 3.33(\mathrm{SD}: \\
0.07)\end{array}$ \\
\hline $\begin{array}{l}\text { ITO:CNT:P3ThEt-g-PANI (10 nm): } \\
\text { PBDT-TIPS-DTNT-DT:PC61BM }\end{array}$ & $\begin{array}{c}\text { Avg: } 9.10(\mathrm{SD}: \\
0.07)\end{array}$ & $\begin{array}{c}\text { Avg: } 53 \text { (SD: } \\
0.40)\end{array}$ & Avg: 0.65 (SD: & $\begin{array}{c}\text { Avg: } 3.13(\mathrm{SD}: \\
0.06)\end{array}$ \\
\hline $\begin{array}{l}\text { ITO:CNT:P3ThEt-g-PANI }(20 \mathrm{~nm}) \text { : } \\
\text { PBDT-DTNT:PC61BM }\end{array}$ & $\begin{array}{c}\text { Avg: } 10.92(\mathrm{SD}: \\
0.06)\end{array}$ & $\begin{array}{c}\text { Avg: } 56(\mathrm{SD}: \\
0.37)\end{array}$ & Avg: $\begin{array}{l}0.66(\mathrm{SD}: \\
0.004)\end{array}$ & Avg: $\begin{array}{c}4.04(\mathrm{SD}: \\
0.05)\end{array}$ \\
\hline $\begin{array}{l}\text { ITO:CNT:P3ThEt-g-PANI (20 nm): } \\
\text { PBDT-TIPS-DTNT-DT:PC61BM }\end{array}$ & Avg: 10.52 (SD: & $\begin{array}{c}\text { Avg: } 54 \text { (SD: } \\
0.39)\end{array}$ & Avg: 0.66 (SD: & $\begin{array}{c}\text { Avg: } 3.75 \text { (SD: } \\
0.05)\end{array}$ \\
\hline $\begin{array}{l}\text { ITO:CNT-g-PDDT:P3ThEt-g-PANI } \\
\text { (10 nm): } \\
\text { PBDT-DTNT:PC61BM }\end{array}$ & $\begin{array}{c}\text { Avg: } 11.18 \text { (SD: } \\
0.06)\end{array}$ & $\begin{array}{c}\text { Avg: } 58 \text { (SD: } \\
0.36)\end{array}$ & $\begin{array}{c}\text { Avg: } 0.67 \text { (SD: } \\
0.005)\end{array}$ & $\begin{array}{c}\text { Avg: } 4.34 \text { (SD: } \\
0.04)\end{array}$ \\
\hline $\begin{array}{l}\text { ITO:CNT-g-PDDT:P3ThEt-g-PANI } \\
\text { (10 nm): } \\
\text { PBDT-TIPS-DTNT-DT:PC61BM }\end{array}$ & Avg: 10.91 (SD: & $\begin{array}{c}\text { Avg: } 55 \text { (SD: } \\
0.38)\end{array}$ & $\begin{array}{cl}\text { Avg: } & 0.66 \text { (SD: } \\
& 0.007)\end{array}$ & $\begin{array}{c}\text { Avg: } 3.96 \text { (SD: } \\
0.05)\end{array}$ \\
\hline $\begin{array}{l}\text { ITO:CNT-g-PDDT:P3ThEt-g-PANI } \\
\text { (20 nm): } \\
\text { PBDT-DTNT:PC61BM }\end{array}$ & Avg: $12.84(\mathrm{SD}:$ & $\begin{array}{c}\text { Avg: } 62 \text { (SD: } \\
0.31)\end{array}$ & $\begin{array}{c}\text { Avg: } \\
0.71 \text { (SD: } \\
0.004)\end{array}$ & $\begin{array}{c}\text { Avg: } 5.65 \text { (SD: } \\
0.03)\end{array}$ \\
\hline $\begin{array}{l}\text { ITO:CNT-g-PDDT:P3ThEt-g-PANI } \\
\text { (20 nm): } \\
\text { PBDT-TIPS-DTNT-DT:PC61BM }\end{array}$ & $\begin{array}{c}\text { Avg: } 11.59 \text { (SD: } \\
0.04)\end{array}$ & $\begin{array}{c}\text { Avg: } 60 \text { (SD: } \\
0.34)\end{array}$ & $\begin{array}{c}\text { Avg: } 0.69 \text { (SD: } \\
0.005)\end{array}$ & $\begin{array}{c}\text { Avg: } 4.80 \text { (SD: } \\
0.04)\end{array}$ \\
\hline
\end{tabular}

Avg average, $S D$ standard deviation.

and $\left.6.82 \mathrm{~mA} / \mathrm{cm}^{2}\right),(49 \%$ and $47 \%)$, and $(0.60 \mathrm{~nm}$ and $0.58 \mathrm{~nm})$ ], and thereby the PCEs $(2.13 \%$ and $1.86 \%$ ) for both PBDT-DTNT and PBDT-TIPSDTNT-DT based solar cells. This may be associated with the more opaque and rougher nature of CNT $(20 \mathrm{~nm})$ thin films, which were dominant to their lower sheet resistance values, i.e., (88-94\% versus 93-96\%), (2.8 nm versus $1.7 \mathrm{~nm})$, and $\left(6.7 \times 10^{5} \Omega /\right.$ sq versus $\left.8.5 \times 10^{5} \Omega / \mathrm{sq}\right)$. The respective $J-V$ curves are depicted in Fig. 5a.

According to a collection of data, the pure CNTs were not the appropriate candidates for being applied instead of conventional PEDOT:PSS HTLs. To tackle this issue, the two $p$-type EBLs were developed on the basis of CNT:P3ThEt-g-PANI and CNT-g-PDDT:P3ThEt-g-PANI thin films with thicknesses of $10 \mathrm{~nm}$ and $20 \mathrm{~nm}$. The J-V curves of these systems as the HTLs in the PBDT-DTNT:PC61BM and PBDT-TIPS-DTNT-DT:PC61BM solar cells are exhibited in Fig. 5b. As 10 thin films of
ITO:CNT:P3ThEt-g-PANI were utilized in the PBDT-DTNT and PBDT-TIPS-DTNT-DT based devices, the results were to some extent better than those reached in the PEDOT:PSS systems. The $J_{\mathrm{sc}}$, $\mathrm{FF}, V_{\mathrm{oc}}$ and PCE parameters were equal to $\left(9.50 \mathrm{~mA} / \mathrm{cm}^{2}\right.$ and $\left.9.10 \mathrm{~mA} / \mathrm{cm}^{2}\right),(54 \%$ and $53 \%)$, $(0.65 \mathrm{~V}$ and $0.65 \mathrm{~V})$ and $(3.33 \%$ and $3.13 \%)$ for the ITO:CNT:P3ThEt-g-PANI (10 nm):PBDTDTNT:PC61BM and ITO:CNT:P3ThEt-g-PANI (10 nm):PBDT-TIPS-DTNT-DT:PC61BM solar cells, respectively. In contrast to the pure CNT systems, herein, via altering the HTL thickness to $20 \mathrm{~nm}$, all photovoltaic properties were improved up to $\left(10.92 \mathrm{~mA} / \mathrm{cm}^{2}\right.$ and $\left.10.52 \mathrm{~mA} / \mathrm{cm}^{2}\right),(56 \%$ and $54 \%)$, $(0.66 \mathrm{~V}$ and $0.66 \mathrm{~V})$ and $(4.04 \%$ and $3.75 \%)$ for the PBDT-DTNT:PC61BM and PBDT-TIPS-DTNTDT:PC61BM devices. The resultant PCEs of $4.04 \%$ and $3.75 \%$ were conspicuously higher than those obtained in the corresponding solar cells, but with the conventional PEDOT:PSS $p$-type EBLs (3.20\% 


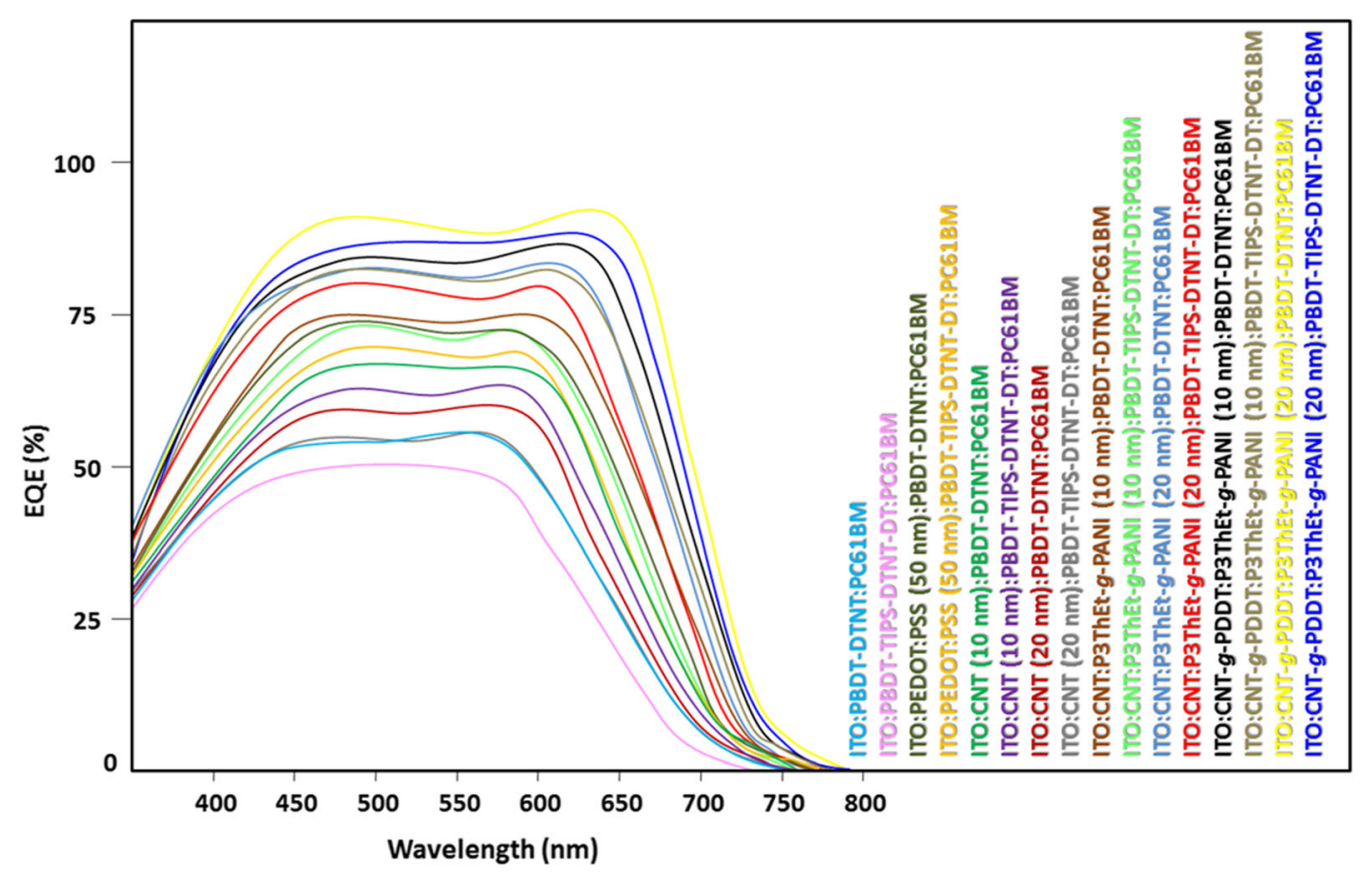

Fig. 6. EQE curves of photovoltaic devices constructed based on BDT-DTNT or PBDT-TIPS-DTNT-DT:PC61BM with the HTLs of PEDOT:PSS, CNT, CNT:P3ThEt- $g$-PANI and CNT- $g$-PDDT:P3ThEt- $g$-PANI versus the ITO only based devices.

and $2.97 \%$ ). Up to here, it could be concluded that the CNT:P3ThEt-g-PANI thin films are appropriate alternatives for the conventional PEDOT:PSS EBLs and a thickness of $20 \mathrm{~nm}$ warrants the superior photovoltaic results.

Going forward, the bared CNTs were replaced by grafted CNTs in the presence of P3ThEt-g-PANI bottlebrushes and, consequently, some interesting data were acquired in both PBDT-DTNT and PBDTTIPS-DTNT-DT based solar cells. As tabulated in Table II, the efficacies of $4.34 \%\left(J_{\mathrm{sc}}=11.18 \mathrm{~mA} /\right.$ $\mathrm{cm}^{2}, \mathrm{FF}=58 \%$, and $\left.V_{\mathrm{oc}}=0.67 \mathrm{~V}\right)$ and $3.96 \%\left(J_{\mathrm{sc}}=\right.$ $10.91 \mathrm{~mA} / \mathrm{cm}^{2}, \quad \mathrm{FF}=55 \%, \quad$ and $\quad V_{\mathrm{oc}}=0.66 \mathrm{~V}$ ) appeared in the ITO:CNT-g-PDDT:P3ThEt-g-PANI (10 nm):PBDT-DTNT:PC61BM and ITO:CNT: P3ThEt-g-PANI (10 nm):PBDT-TIPS-DTNT-DT: PC61BM devices, respectively. Compared to the corresponding systems, but without grafting of CNT precursors, the performances were multiplied by 1.30 (PBDT-DTNT) and 1.26 (PBDT-TIPS-DTNTDT). In fact, the CNT-g-PDDT:P3ThEt-g-PANI HTLs possessing the RMS $=1.1 \mathrm{~nm}$, the sheet resistance of $8.3 \times 10^{4} \Omega / \mathrm{sq}$, and the transmittance ranged in 84-90\% acted better than all other systems. This phenomenon could be assigned to an interconnected network composed of CNTs grafted with the polythiophenic agents and on the other side, the polythiophenic backbones covered with the conjugated PANI side brushes.

Raising trend of photovoltaic and physical properties eventually peaked at the $20 \mathrm{~nm}$ thin films of CNT-g-PDDT:P3ThEt-g-PANI, demonstrating the largest PCE values of $5.65 \%$ and $4.80 \%$ in the BDT-DTNT and PBDT-TIPS-DTNT-DT based devices, respectively. As expected, the $J_{\mathrm{sc}}, \mathrm{FF}$, and
$V_{\text {oc }}$ parameters were also the largest in the mentioned systems. The ITO:CNT-g-PDDT:P3ThEt-gPANI (20 nm):PBDT-DTNT:PC61BM and ITO: CNT-g-PDDT:P3ThEt-g-PANI $\quad(20 \mathrm{~nm}): P B D T-$ TIPS-DTNT-DT:PC61BM solar cells reflected the pair data of $\left(12.84 \mathrm{~mA} / \mathrm{cm}^{2}\right.$ and $\left.11.59 \mathrm{~mA} / \mathrm{cm}^{2}\right)$, $(62 \%$ and $60 \%)$, and $(0.71 \mathrm{~V}$ and $0.69 \mathrm{~V})$, respectively. It could be anticipated that an interconnected network in the CNT-g-PDDT:P3ThEt-g-PANI thin films had better opportunity in the thicker films of $20 \mathrm{~nm}$ to further grow and thus reflect the smoothest morphology (RMS $=1.0 \mathrm{~nm})$ with the smallest sheet resistance $\left(=2.2 \times 10^{4} \Omega / \mathrm{sq}\right)$. Although an enhancement in the thickness of CNT HTLs decreased the sheet resistance (from $8.5 \times 10^{5} \Omega$ / sq to $6.7 \times 10^{5} \Omega / \mathrm{sq}$ ), it significantly elevated the thin film RMS (from $1.7 \mathrm{~nm}$ to $2.8 \mathrm{~nm}$ ). It could be comprehended that the CNTs were roughly arranged in the thicker HTLs. In a forward step, the incorporation of P3ThEt-g-PANI bottlebrushes with polythiophenic backbones and conjugated rod PANI brushes into the HTLs led to more homogeneous thin films (RMS $=1.3-1.4 \mathrm{~nm})$ with the lower sheet resistances $\left(1.1-4.9 \times 10^{5} \Omega / \mathrm{sq}\right)$. A smoother morphology in the thicker HTL $(20 \mathrm{~nm}$ versus $10 \mathrm{~nm}$ ) could be a fingerprint of the fact that the P3ThEt-g-PANI bottlebrushes and CNT precursors had a higher degree of freedom to prepare a homogeneous conductive network. On the other side, the grafting of CNTs with the polythiophenic oligomers (PDDT chains) provided a better opportunity for the fabrication of more homogeneous network between the CNT-g-PDDT and P3ThEt-gPANI constituents. Hence, the lowest sheet resistance $\left(2.2 \times 10^{4} \Omega / \mathrm{sq}\right)$ and the smoothest 
morphology $(\mathrm{RMS}=1.0 \mathrm{~nm})$ were reached for the CNT-g-PDDT:P3ThEt-g-PANI having a thickness of $20 \mathrm{~nm}$. The photovoltaic results, in particular the PCE values, approved this progressing trend in the HTL quality.

The samples were also analyzed by means of the external quantum efficiency (EQE) and the recorded spectra are depicted in Fig. 6 for all fabricated solar cells. Notably, the external quantum performances were in complete accord with the previous results obtained in the physical (Figs. 3 and 4 and Table I) and photovoltaic (Fig. 5 and Table II) sections. The best peak values about $91-93 \%$ and $87-89 \%$ were determined at $632 \mathrm{~nm}$ and $625 \mathrm{~nm}$ for the ITO:CNT-g-PDDT:P3ThEt-g-PANI (20 nm):PBDTDTNT:PC61BM and ITO:CNT-g-PDDT:P3ThEt-gPANI (20 nm):PBDT-TIPS-DTNT-DT:PC61BM solar cells, respectively. Similar systems to the above-mentioned photovoltaics, but with a thickness of $10 \mathrm{~nm}$, also led to the proper EQE results, i.e., $84-87 \%$ at $618 \mathrm{~nm}$ and $81-82 \%$ at $607 \mathrm{~nm}$ detected for the PBDT-DTNT and PBDT-TIPSDTNT-DT based devices, respectively. Thereafter, the nanocomposites of ungrafted CNT precursors in the presence of P3ThEt-g-PANI bottlebrushes having the thicknesses of $20 \mathrm{~nm}$ and $10 \mathrm{~nm}$ reflected the acceptable EQE data (Fig. 6: 81-83\% at $607 \mathrm{~nm}$ for the ITO:CNT:P3ThEt-g-PANI (20 nm):PBDTDTNT:PC61BM, $79-80 \%$ at $600 \mathrm{~nm}$ for the ITO: CNT:P3ThEt-g-PANI (20 nm):PBDT-TIPS-DTNTDT:PC61BM, 75-76\% at $594 \mathrm{~nm}$ for the ITO:CNT: P3ThEt-g-PANI (10 nm):PBDT-DTNT:PC61BM and $71-73 \%$ at $582 \mathrm{~nm}$ for the ITO:CNT:P3ThEt$g$-PANI (10 nm):PBDT-TIPS-DTNT-DT:PC61BM solar cells). As previously discussed, the functions of pure CNT thin films as the $p$-type EBLs, in particular with a thickness of $20 \mathrm{~nm}$, were worse than the conventional PEDOT:PSS HTLs in both PBDT-DTNT and PBDT-TIPS-DTNT-DT based devices. The EQE curves reported in Fig. 6 also approved the results in question. By large, although the pure CNTs were not appropriate choices for the replacement of conventional PEDOT:PSS HTLs, their derivatives, especially those grafted with the polythiophenic agents and blended with the rod-rod full-conjugated bottlebrushes, acted their role even better than the PEDOT:PSS $p$-type EBLs.

\section{CONCLUSIONS}

The replacement of PEDOT:PSS with the pure CNT films did not afford the high-quality characteristics $(\mathrm{PCE}=1.86-2.84 \%)$. To tackle this issue, the two $p$-type EBLs were developed on the basis of CNT:P3ThEt-g-PANI and CNT-g-PDDT:P3ThEt-gPANI thin films with the thicknesses of $10 \mathrm{~nm}$ and $20 \mathrm{~nm}$. Among the modified HTLs, the CNT-gPDDT:P3ThEt-g-PANI $(20 \mathrm{~nm})$ thin films represented the best photovoltaic properties of $\left(12.84 \mathrm{~mA} / \mathrm{cm}^{2}\right.$ and $\left.11.59 \mathrm{~mA} / \mathrm{cm}^{2}\right),(62 \%$ and $60 \%)$, $(0.71 \mathrm{~V}$ and $0.69 \mathrm{~V})$ and $(5.65 \%$ and $4.80 \%)$ in the
BDT-DTNT and PBDT-TIPS-DTNT-DT based devices, respectively. The CNT-g-PDDT:P3ThEt-gPANI thin films, possessing an interconnected network composed of grafted-CNTs and P3ThEt-gPANI bottlebrushes, were appropriate alternatives for the conventional PEDOT:PSS HTLs and warranted the superior photovoltaic results by the smooth morphologies (root mean square $=1.0$ $1.1 \mathrm{~nm})$ and low sheet resistances (2.2$\left.8.3 \times 10^{4} \Omega / \mathrm{sq}\right)$. Similar HTLs but without grafting of CNT precursors also led to the proper data (3.13$4.04 \%$ ). The networks of ungrafted-CNTs and polythiophene-grafted-CNTs with conducting P3ThEt-gPANI bottlebrushes could compete with the conventional PEDOT:PSS films to be incorporated as $p$ type EBLs in the organic solar cells.

\section{ACKNOWLEDGMENTS}

Project funded by China Postdoctoral Science Foundation, the Electronic Fence System project, the Project of FDCT, and the Project of the Macao Foundation.

\section{ELECTRONIC SUPPLEMENTARY MATERIAL}

The online version of this article (https://doi.org/ 10.1007/s11664-019-07852-w) contains supplementary material, which is available to authorized users.

\section{REFERENCES}

1. C.J. Brabec, N.S. Sariciftci, and J.C. Hummelen, $A d v$. Funct. Mater. 11, 15 (2001).

2. H. Hoppe and N.S. Sariciftci, J. Mater. Res. 19, 194 (2004).

3. G. Yu and A.J. Heeger, J. Appl. Phys. 78, 4510 (1995).

4. J.U. Lee, A. Cirpan, T. Emrick, T.P. Russell, and W.H. Jo, J. Mater. Chem. 19, 1483 (2009).

5. H. Hoppe and N.S. Sariciftci, J. Mater. Chem. 16, 45 (2006).

6. G. Dennler, M.C. Scharber, and C.J. Brabec, Adv. Mater. 21, 1323 (2009).

7. S.A. Carter, M. Angelopoulos, S. Karg, P.J. Brock, and J.C. Scott, Appl. Phys. Lett. 70, 2067 (1997).

8. T.M. Brown, J.S. Kim, R.H. Friend, F. Cacialli, R. Daik, and W.J. Feast, Appl. Phys. Lett. 75, 1679 (1999).

9. S. Khodabakhsh, B.M. Sanderson, J. Nelson, and T.S. Jones, Adv. Funct. Mater. 16, 95 (2006).

10. H. Lian, N. Jun, A. Bolag, A. Hexig, N. Gerile, O. Tegus, and S. Lin, Solid State Phenom. 288, 113 (2019).

11. M.S. Ramasamy, K.Y. Ryu, J.W. Lim, A. Bibi, H. Kwon, J.E. Lee, D.H. Kim, and K. Kim, Nanomaterials 9, 1328 (2019).

12. A. Rana, A. Kumar, S. Chand, and R.K. Singh, J. Appl. Phys. 125, 053102 (2019).

13. The material information is available in the H.C. Starck web site http://www.hcstarck.com/.

14. W.H. Kim, A.J. Mäkinen, N. Nikolov, R. Shashidhar, H. Kim, and Z.H. Kafafi, Appl. Phys. Lett. 80, 3844 (2002).

15. H. Yan, P. Lee, N.R. Armstrong, A. Graham, G.A. Evmenenko, P. Dutta, and T.J. Marks, J. Am. Chem. Soc. 127, 3172 (2005).

16. J. Van De Lagemaat, T.M. Barnes, G. Rumbles, S.E. Shaheen, T.J. Coutts, C. Weeks, I. Levitsky, J. Peltola, and P. Glatkowski, Appl. Phys. Lett. 88, 233503 (2006). 
17. K. Norrman, M.V. Madsen, S.A. Gevorgyan, and F.C. Krebs, J. Am. Chem. Soc. 132, 16883 (2010).

18. A.W. Hains and T.J. Marks, Appl. Phys. Lett. 92, 023504 (2008).

19. M. Kemerink, S. Timpanaro, M.M. De Kok, E.A. Meulenkamp, and F.J. Touwslager, J. Phys. Chem. B 108, 18820 (2004).

20. L.M. Chen, Z. Xu, Z. Hong, and Y. Yang, J. Mater. Chem. 20, 2575 (2010).

21. M.S. White, D.C. Olson, S.E. Shaheen, N. Kopidakis, and D.S. Ginley, Appl. Phys. Lett. 89, 143517 (2006).

22. S.K. Hau, H.L. Yip, N.S. Baek, J. Zou, K. O'Malley, and A.K.Y. Jen, Appl. Phys. Lett. 92, 225 (2008).

23. Z. Zhao, Q. Wu, F. Xia, X. Chen, Y. Liu, W. Zhang, J. Zhu, S. Dai, and S. Yang, ACS Appl. Mater. Interfaces 7, 1439 (2015).

24. L. Lu, T. Xu, I.H. Jung, and L. Yu, J. Phys. Chem. C 118, 22834 (2014).

25. Y. Jiang, S. Xiao, B. Xu, C. Zhan, L. Mai, X. Lu, and W. You, ACS Appl. Mater. Interfaces 8, 11658 (2016).

26. J. Kim, H. Kim, G. Kim, H. Back, and K. Lee, ACS Appl. Mater. Interfaces 6, 951 (2014).

27. H.T. Chien, F. Pilat, T. Griesser, H. Fitzek, P. Poelt, and B. Friedel, ACS Appl. Mater. Interfaces 10, 10102 (2018).

28. W.J. Ke, G.H. Lin, C.P. Hsu, C.M. Chen, Y.S. Cheng, T.H. Jen, and S.A. Chen, J. Mater. Chem. 21, 13483 (2011).

29. W. Zhao, L. Ye, S. Zhang, B. Fan, M. Sun, and J. Hou, Sci. Rep. 4, 6570 (2014).
30. J.W. Jung, J.U. Lee, and W.H. Jo, J. Phys. Chem. C 114, 633 (2009).

31. W.J. Bae, K.H. Kim, Y.H. Park, and W.H. Jo, Chem. Commun. 22, 2768 (2003).

32. W.J. Bae, K.H. Kim, W.H. Jo, and Y.H. Park, Macromolecules 38, 1044 (2005).

33. Y. Sun, S.C. Chien, H.L. Yip, Y. Zhang, K.S. Chen, D.F. Zeigler, F.C. Chen, B. Lin, and A.K.Y. Jen, Chem. Mater. 23, 5006 (2011).

34. E. Kymakis, M.M. Stylianakis, G.D. Spyropoulos, E. Stratakis, E. Koudoumas, and C. Fotakis, Sol. Energy Mater. Sol. Cells 96, 298 (2012).

35. C.T. Smith, R.W. Rhodes, M.J. Beliatis, K.D.G. Imalka Jayawardena, L.J. Rozanski, C.A. Mills, and P.S.R. Silva Appl. Phys. Lett. 105, 129 (2014).

36. R. Sarvari, M. Akbari-Alanjaraghi, B. Massoumi, Y. BeygiKhosrowshahi, and S. Agbolaghi, New J. Chem. 41, 6371 (2017).

37. B. Massoumi, R. Sarvari, and S. Agbolaghi, Int. J. Polym. Mater. Polym. Biomater. 67, 808 (2018).

38. M. Wang, X. Hu, P. Liu, W. Li, X. Gong, F. Huang, and Y. Cao, J. Am. Chem. Soc. 133, 9638 (2011).

39. J. Tong, L. An, J. Li, P. Zhang, P. Guo, C. Yang, Q. Su, X. Wang, and Y. Xia, J. Macromol. Sci. A 54, 176 (2017).

Publisher's Note Springer Nature remains neutral with regard to jurisdictional claims in published maps and institutional affiliations. 